\title{
VARIABILIDADE DE FÓSFORO E POTÁSSIO DISPONÍVEL NO SOLO EM DIFERENTES ESPAÇAMENTOS DE PLANTIO DE TAXI BRANCO (Tachigali vulgaris)
}

\author{
Tainah Silva Narducci ${ }^{1}$, Jorge Alberto Gazel Yared ${ }^{2}$, Silvio Brienza Júnior ${ }^{3}$, Veríssimo Cesar Sousa da \\ Silva $^{4}$, Shislene Rodrigues de Souza ${ }^{5}$
}

\section{RESUMO:}

O objetivo deste trabalho foi avaliar a variabilidade vertical e horizontal do teor de fósforo e potássio do solo de um plantio de taxi-branco (Tachigali vulgaris) com aproximadamente sete anos e meio de idade, em três espaçamentos, localizado em Dom Eliseu, PA. As parcelas amostrais foram dispostas em blocos ao acaso com quatro repetições, nas quais foram escolhidos, aleatoriamente, 16 pontos de coleta do solo em quatro profundidades. As amostras de solo coletadas foram transportadas para o laboratório de solos da Embrapa Amazônia Oriental, onde foram realizadas as análises químicas. Concluiu-se que nos espaçamentos mais adensados, houve diferença significativa entre as profundidades, para o teor de fósforo e potássio, mostrando-se maior teor na superfície do solo. Porém, em profundidade total apresentou diferença significativa entre os espaçamentos, mais adensado e menos adensado.

Palavras-chave: Densidade, fertilidade do solo, profundidade.

\section{VARIABILITY OF PHOSPHORUS AND POTASSIUM AVAILABLE IN DIFFERENT SOIL SPACINGS OF TACHI-BRANCO (Tachigali vulgaris) PLANTATION}

\begin{abstract}
:
The objective of this work was to evaluate the vertical and horizontal variability of the content of phosphorus and potassium on the soil of a "tachi-branco" (Tachigali vulgaris) plantation, with approximately seven and a half years old, in three spacings, located in Dom Eliseu, PA. Sample plots were arranged in randomizes blocks with four replications, in which 16 soil collecting points were randomly selected at four depth. The collected soil samples were transported to the soil laboratory of the Agricultural Research Corporation of Belém/Pará "Embrapa Amazônia Oriental", where the chemical analyzes was carried out. It was concluded that in the denser spacings, there was a significant difference between the depths for the

\footnotetext{
${ }^{1}$ Graduação em Engenharia Ambiental-PUC/GO, Mestre em Ciências Ambientais-UFPA; Secretaria de Estado de Meio Ambiente e Sustentabilidade/SEMAS-PA; tainahnarducci@hotmail.com

${ }^{2}$ Graduação em Engenharia Florestal-UFPR, Doutor em Ciências Florestais-UFV; Embrapa Amapá; jagyared@ gmail.com

${ }^{3}$ Graduação em Engenharia Florestal-USP, Doutor em Agricultura Tropical-George August University of Goettingen, Alemanha; Embrapa Amazônia Oriental; silvio.brienza@embrapa.br

4 Graduação em Engenharia Florestal-UFRA, Doutorando em Ciências Ambiental-UFPA; Universidade Federal do Pará; verissimo_cesar@yahoo.com.br

${ }^{5}$ Graduação em Engenharia Florestal-UFRA; Mestre em Ciências Florestais-UFRA; Instituto de Desenvolvimento Florestal e da Biodiversidade do Estado do Pará/Ideflor-bio; leneforest@gmail.com
} 
Revista Agri-Environmental Sciences, Palmas-TO, v. 5, e019006, 2019

phosphorus and potassium content, showing a higher proportion in the soil surface. However, in relation to total depth, results showed significant difference between the spacings, such as thicker and less thick.

Keywords: Density, soil fertility, depth. 


\section{INTRODUÇÃO}

A diversidade biológica e ecológica da região Amazônica se destaca não só pelas suas áreas exuberantes, mas também pela potencialidade de uso dos recursos resguardados nas mais diversas formas. Para tanto, entender a forma como cada sistema se apresenta no ambiente é fundamental para conduzir arranjos que reproduzam fonte de matéria prima.

O taxi-branco (Tachigali vulgaris L.F. \& H.C) é uma leguminosa arbórea nativa da Amazônia que se apresenta como fonte de biomassa florestal de excelente qualidade, além de ter uma adaptação boa para os solos pobres e ácidos que ocorrem na Amazônia (Brienza Júnior et al., 2011). A espécie que integra a família das Fabaceae tem se destacado como fonte promissora para produção de energia, com características comparáveis ao Eucalyptus sp. L'Hér.

A atratividade de uso múltiplo, associados aos benefícios ecológicos, também tem se mostrado promissores para o plantio da espécie. Por outro lado, conhecer os gradientes edáficos e suas diversas aplicações de manejo pode auxiliar na inclusão da espécie em arranjos produtivos mais adequados ao ambiente e menos oneroso para o estabelecimento da cultura (Brienza júnior et al., 2011). A aplicação superficial de adubos, a condição de cobertura e revolvimento do solo interfere na dinâmica de nutrientes, especialmente de fósforo (Nolla e Anghinoni, 2006).

$\mathrm{O}$ fósforo é um elemento essencial à vida e tem uma distribuição muito irregular na natureza, de modo a torná-lo limitante a produção agrícola em muitas regiões (Klein e Agne, 2012). Para a produção de espécies florestais não é diferente, pois a disponibilidade do elemento em algumas condições é inexpressiva ou desfavorável, o que pode colocar em risco a manutenção de povoamentos florestais inteiros. De acordo com Barros et al. (2005), as maiores respostas na produção florestal são obtidas pela aplicação de fósforo na época de plantio. $\mathrm{O}$ fósforo é decisivo no metabolismo das plantas, desempenhando papel importante na transferência de energia da célula, na respiração e na fotossíntese, entre outras funções ligadas aos componentes estruturais de desenvolvimento da planta (Grantet al., 2001).

O potássio é o segundo nutriente para o qual se conseguem respostas mais elevadas para florestas plantadas (Barros et al., 2005). O nutriente age livre na planta e não forma compostos como acontece com o fósforo. Uma função importante diz respeito às reações enzimáticas. Em razão dessa característica, permanece quase que totalmente na forma iônica nos tecidos vegetais (Foloni, et al., 2012). Na condição da cultura estar em senescência ou em manejo, há rápida liberação do potássio contido nos restos vegetais para o solo com a ação das chuvas, sem que haja decomposição biológica expressiva dos tecidos (Rosolem et al., 2003).

Neste sentido, o objetivo deste trabalho foi analisar os teores de fósforo e potássio do solo sobre um plantio de $T$. vulgaris entre as diferentes profundidades de cada espaçamento, e entre os diferentes espaçamentos de cada profundidade.

\section{MATERIAL E MÉTODOS}

O estudo foi desenvolvido em um plantio de T. vulgaris com sete anos e meio de idade, localizado na Fazenda Gênesis, Dom Eliseu, Pará, km 49, BR222 , latitude $04^{\circ} 33^{\prime} 10^{\prime}$ ' $\mathrm{S}$ e longitude $47^{\circ} 49^{\prime} 0^{\prime}$ ' $\mathrm{W}$.

O clima da cidade de Dom Eliseu é caracterizado como mesotérmico úmido, com temperatura média anual por volta de $25^{\circ} \mathrm{C}$, regime pluviométrico, frequentemente, entre $2.250 \mathrm{~mm}$ e $2.500 \mathrm{~mm}$, e chuvas regulares, porém sua maior concentração (em torno de $80 \%$ ) está entre os meses de janeiro a junho (Fapespa, 2016).

A vegetação do município corresponde à floresta Amazônica, do subtipo Floresta densa da subregião dos altos platôs do Pará e Maranhão, Floresta densa de planície aluvial e densa dos terraços (Fapespa, 2016). No município, os tipos de solos existentes incluem o Latossolo Amarelo, textura muito argilosa; o Latossolo Amarelo, textura argilosa; Concrecionário Laterítico; Latossolo 
Amarelo, textura média e Areias Quartzosas, em associações (Fapespa, 2016). Antes do plantio das mudas foram aplicados nas covas $200 \mathrm{~g}$ de NPK na proporção 10:28:20, e após o plantio, as adubações de cobertura nas plantas foram feitas no início e no final do período chuvoso com 75 g de NPK (Sousa, 2011).

A coleta das amostras foi realizada em agosto de 2013, em uma área de plantio homogêneo com $18.000 \mathrm{~m}^{2}$, a qual foi dividida em seis blocos ao acaso de 50 × $60 \mathrm{~m}$, em três espaçamentos. Nos blocos foram instaladas parcelas de $50 \times 30 \mathrm{~m}$ com quatro repetições para cada espaçamento de plantio ( $4 \times 2 \mathrm{~m}$; $4 \times 3 \mathrm{~m} \mathrm{e} 4 \times 4 \mathrm{~m})$. Em cada parcela $(50 \times 30 \mathrm{~m})$ foram escolhidos, aleatoriamente, 16 pontos de coleta de solo. Os solos coletados nos 16 pontos foram misturados e retiradas quatro amostras compostas em quatro profundidades: $0-5 \mathrm{~cm} ; 5-10 \mathrm{~cm} ; 10-20$ $\mathrm{cm} ; 20-30 \mathrm{~cm}$. Assim, foram obtidas 192 amostras de solo que posteriormente foram encaminhados para o Laboratório de Solos da Embrapa Amazônia Oriental - Belém/PA, onde se submeteu à análise química de fósforo e potássio dos produtos e onde o procedimento laboratorial para análise química do fósforo e potássio foi realizado, conforme metodologias descritas por Silva et al. (1998).

Para análise estatística, foram avaliadas as médias dos elementos para cada profundidade e para a profundidade total, nos três espaçamentos. As médias obtidas foram submetidas à análise de variância (ANOVA) para averiguar se os diferentes espaçamentos do plantio de T. vulgaris e as diferentes profundidades influenciavam no teor dos elementos no solo. A análise estatística foi realizada no Microsoft Excel 2013 e no software PAST.

\section{RESULTADOS E DISCUSSÃO}

Os valores das médias das 16 amostras compostas, de cada profundidade, nos três espaçamentos de plantio de T. vulgaris, e a análise de variância entre as profundidades são apresentados na tabela 1. Observou-se que os teores de fósforo e potássio apresentaram diferenças significativas entre as profundidades nos espaçamentos 4 x 2 e 4 x $3 \mathrm{~m}$.

$\mathrm{Na}$ Tabela 1, constatou-se que no espaçamento $4 \times 2$, o teor de fósforo foi superior na profundidade $0-5 \mathrm{~cm}$ que nas profundidades de 5-10 e 20-30 cm. Nesse mesmo espaçamento, o potássio foi maior até os $5 \mathrm{~cm}$ de profundidade quando comparado com as coletas mais profundas (10-20 e 20-30 cm). Já no espaçamento 4 × $3 \mathrm{~m}$, os teores de fósforo e o potássio foram superiores até os $10 \mathrm{~cm}$ de profundidade que na camada de 10 a $30 \mathrm{~cm}$.

Tabela 1. Médias do teor de $\mathrm{P}\left(\mathrm{mg} \mathrm{kg}^{-1}\right)$ e $\mathrm{K}\left(\mathrm{mg} \mathrm{kg}^{-1}\right)$ nos espaçamentos de plantio e análise de variância (ANOVA) das médias entre as profundidades.

\begin{tabular}{|c|c|c|c|c|c|}
\hline \multicolumn{6}{|c|}{ Profundidades } \\
\hline $\mathbf{P}$ & $0-5 \mathrm{~cm}$ & $5-10 \mathrm{~cm}$ & $10-20 \mathrm{~cm}$ & $20-30 \mathrm{~cm}$ & ANOVA \\
\hline $4 \times 2 \mathrm{~m}$ & $10,38 \mathrm{a}$ & $5,44 \mathrm{~b}$ & $6,56 \mathrm{ab}$ & $4,75 \mathrm{~b}$ & $\mathrm{~F}=4,670 ; \mathrm{p}<0,005$ \\
\hline $4 \times 3 \mathrm{~m}$ & $8,44 \mathrm{a}$ & 8,19 a & $3,75 b$ & $3,38 \mathrm{~b}$ & $\mathrm{~F}=10,937 ; \mathrm{p}<$ \\
\hline $4 \times 4 \mathrm{~m}$ & 6,06 a & $4,19 \mathrm{a}$ & $5,38 \mathrm{a}$ & $2,81 \mathrm{a}$ & $F=1,954 ; p<0,131$ \\
\hline \multicolumn{6}{|l|}{$\mathbf{K}$} \\
\hline $4 \times 2 \mathrm{~m}$ & $33,50 \mathrm{a}$ & $26,94 a b$ & $21,63 \mathrm{~b}$ & $21,69 \mathrm{~b}$ & $F=6,760 ; p<0,001$ \\
\hline $4 \times 3 \mathrm{~m}$ & $76,63 \mathrm{a}$ & $66,50 \mathrm{a}$ & $42,88 \mathrm{~b}$ & $36,13 \mathrm{~b}$ & $\mathrm{~F}=10,560 ; \mathrm{p}<$ \\
\hline $4 \times 4 \mathrm{~m}$ & $63,81 \mathrm{a}$ & $49,13 \mathrm{a}$ & 60,63 a & $38,13 \mathrm{a}$ & $F=0,923 ; p<0,435$ \\
\hline
\end{tabular}

Médias seguidas pela mesma letra na linha não diferem entre si. Teste de Tukey $(\mathrm{p}<0,05)$. / Averages followed by the same letter in the row do not differ statistically. Tukey test $(\mathrm{p}<0.05)$. 
Foi analisado também a ANOVA das médias do teor de fósforo e potássio de cada espaçamento de plantio de T. vulgaris, nas profundidades avaliadas e na profundidade total (Tabela 2). Em todas as profundidades de solo coletadas o espaçamento 4 x 2 apresentou valores superiores ou iguais de fósforo e nitrogênio quando comparado aos espaçamentos $4 \mathrm{x}$ 3 e 4 x 4. Os teores de fósforo na camada total e até os $5 \mathrm{~cm}$, e teor de potássio de 10 a $30 \mathrm{~cm}$ foram maiores no espaçamento de 4 x 2 diferente do observado no 4 x 4 , não diferindo do espaçamento 4 x 3. Já o espaçamento 4 x 2 apresentou maiores teores de fósforo na camada de $5-10 \mathrm{~cm}$ e de potássio na camada de 0-5 cm, quando comparado ao espaçamento de $4 \times 3$, mas não diferiu do $4 \times 4$. Para o potássio de $5-10 \mathrm{~cm}$ e potássio total, no espaçamento $4 \times 2$ foi superior aos de $4 \times 3$ e 4 x 4 .

Há uma estreita relação entre transporte, absorção de nutrientes, umidade do solo e por consequência de produção florestal. A relação entre produtividade e acúmulo de nutrientes é mais estreita em solos de fertilidade mais baixa. Em sítios com condições que permitam elevada produtividade florestal, há forte pressão sobre os recursos do solo, isto é, água e nutrientes. (Barros et al. 2005). O nível de nutriente fósforo e potássio, identificado em cada ponto pode ser reflexo da quantidade do NPK aplicado na cova durante o procedimento de adubação realizado antes do plantio. Este fato, também, pode explicar o comportamento identificado neste estudo quanto à disponibilidade de fósforo e potássio no solo por ocasiões das coletas nas diferentes profundidades.

No geral, foi verificado que existe uma estreita relação entre os dois nutrientes com as profundidades; é uma relação negativa entre as duas grandezas estudadas, ou seja, os níveis de fósforo e potássio nas profundidades mais superficiais apresentaram uma menor diferença significativa, comparado com os níveis mais profundos.

A maior diferença no teor de fósforo entre a profundidade de $0-5 \mathrm{~cm}$ e a $20-30$ foi de $5,63 \mathrm{mg} \mathrm{kg}^{-}$ ${ }^{1}$, observada no espaçamento 4 x 2 ; já para o potássio essa diferença foi de $40,50 \mathrm{mg} \mathrm{kg}^{-1}$ no espaçamento 4 $x$ 3. Isso talvez seja pela ciclagem de nutrientes (Santos e Tomm, 2003), assim como os corretivos aplicados nas camadas superficiais e à não mobilização de fertilizantes (Demaria et al., 1999).

A absorção pelas raízes das plantas dependente da disponibilidade de fósforo no solo e do volume de adubação (Anghinoni, 1992; Model e Anghinoni, 1992; Klepker e Anghinoni, 1995). Essa absorção de suprimentos pode sofrer variação devido o fósforo apresentar pouco teor disponível em solos oxídicos (Novais et al., 2007), além de pouca mobilidade no solo (Barber, 1984). Este dado pode ser confirmado por Falcão e Escosteguy (2006) em Latossolo Vermelho alumino férrico que encontraram diferença estatística nos teores de fósforo, potássio e matéria orgânica, entre as camadas de solo, havendo decréscimo nos teores com o incremento na profundidade de amostragem. Já os resultados do fósforo e potássio foram semelhantes aos obtidos por outros pesquisadores (Schlindwein e Anghinoni, 2000; Anghinoni e Salet, 1998; Carvalho et al., 2002).

Quando se analisa o espaçamento em relação ao teor dos nutrientes para fósforo, constatou-se uma relação inversamente proporcional; quanto maior o espaçamento menor $o$ teor desse nutriente, corroborado no estudo de Pereira (1990), onde este autor constatou aumento do acúmulo de nutrientes por unidade de área com o aumento da densidade. Isso pode estar relacionado à maior quantidade deste nutriente em detrimento de uma maior densidade populacional, ou seja, espaçamentos menores possuem maior número de árvores por hectare e consequentemente maior número de folhas produzidas, contribuindo para maior acúmulo sobre o solo (Silveira et al., 2014) e, na medida em que o material decíduo vai se decompondo, os nutrientes nele contido são liberados dando sequência à ciclagem de nutrientes (Schumacher et al., 2004; Laclau et al., 2010). 
Tabela 2. Médias do teor de $\mathrm{P}\left(\mathrm{mg} \mathrm{kg}^{-1}\right)$ e $\mathrm{K}\left(\mathrm{mg} \mathrm{kg}^{-1}\right)$ nas profundidades avaliadas e análise de variância (ANOVA) das médias entre os espaçamentos de plantio.

\begin{tabular}{ccccc}
\hline \multicolumn{5}{c}{ Espaçamentos } \\
\hline $\mathbf{4}$ & $\mathbf{4} \mathbf{2} \mathbf{~ m}$ & $\mathbf{4 \times 3} \mathbf{~ m}$ & $\mathbf{4 \times 4} \mathbf{~ m}$ & ANOVA \\
\hline Prof. 0-5 cm & $10,38 \mathrm{a}$ & $8,44 \mathrm{ab}$ & $6,06 \mathrm{~b}$ & $\mathrm{~F}=3,191 ; \mathrm{p}<0,051$ \\
Prof. 5-10 cm & $5,44 \mathrm{a}$ & $8,19 \mathrm{~b}$ & $4,19 \mathrm{a}$ & $\mathrm{F}=10,586 ; \mathrm{p}<0,000$ \\
Prof. 10-20 cm & $6,56 \mathrm{a}$ & $3,75 \mathrm{a}$ & $5,38 \mathrm{a}$ & $\mathrm{F}=1,034 ; \mathrm{p}<0,364$ \\
Prof. 20-30cm & $4,75 \mathrm{a}$ & $3,38 \mathrm{a}$ & $2,81 \mathrm{a}$ & $\mathrm{F}=3,094 ; \mathrm{p}<0,055$ \\
Prof. total & $6,78 \mathrm{a}$ & $5,94 \mathrm{ab}$ & $4,61 \mathrm{~b}$ & $\mathrm{~F}=3,898 ; \mathrm{p}<0,022$ \\
\hline K & & & \\
\hline Prof. 0-5 cm & $33,50 \mathrm{a}$ & $76,63 \mathrm{~b}$ & $63,81 \mathrm{ab}$ & $\mathrm{F}=6,258 ; \mathrm{p}<0,004$ \\
Prof. 5-10 cm & $26,94 \mathrm{a}$ & $66,50 \mathrm{~b}$ & $49,13 \mathrm{~b}$ & $\mathrm{~F}=12,377 ; \mathrm{p}<0,000$ \\
Prof. 10-20 cm & $21,63 \mathrm{a}$ & $42,88 \mathrm{ab}$ & $60,63 \mathrm{~b}$ & $\mathrm{~F}=3,106 ; \mathrm{p}<0,055$ \\
Prof. 20-30cm & $21,69 \mathrm{a}$ & $36,13 \mathrm{ab}$ & $38,13 \mathrm{~b}$ & $\mathrm{~F}=4,526 ; \mathrm{p}<0,016$ \\
Prof. total & $25,94 \mathrm{a}$ & $55,53 \mathrm{~b}$ & $52,92 \mathrm{~b}$ & $\mathrm{~F}=15,746 ; \mathrm{p}<0,000$ \\
\hline
\end{tabular}

Médias seguidas pela mesma letra na linha não diferem entre si estatisticamente. Teste de Tukey $(\mathrm{p}<0,05)$. / Averages followed by the same letter in the row do not differ statistically. Tukey test $(\mathrm{p}<0.05)$.

O teor de potássio verificado, apesar de apresentar diferença significativa em todas as profundidades entre os espaçamentos, não apresentou uma relação positiva ou negativa, diferenciando-se de outros trabalhos pesquisados na literatura, como Leite (1998) que concluiu que a maior densidade populacional provoca maior concentração de potássio em folhas e galhos e também no solo devido a decomposição dessas folhas, influenciando positivamente na ciclagem de nutriente.

\section{CONCLUSÃO}

Os espaçamentos mais adensados ( 4 × $2 \mathrm{~m} \mathrm{e} 4$ x $3 \mathrm{~m}$ ) apresentaram diferenças significativas nos teores de fósforo e potássio entre as profundidades. A superfície do solo mostrou maior teor de fósforo e potássio em relação às camadas mais profundas.

A profundidade total mostrou diferença significativa entre os espaçamentos mais adensado (4 x $2 \mathrm{~m}$ ) e menor adensado (4 x $4 \mathrm{~m}$ ) para o fósforo, com maior teor no espaçamento com mais indivíduo arbóreo. Para o potássio, a profundidade total mostrou diferença significativa entre os espaçamentos mais adensado ( $4 \times 2 \mathrm{~m}$ ) e os menos adensados ( 4 × $3 \mathrm{~m} \mathrm{e}$ $4 \times 4 \mathrm{~m}$ ), com menor teor no espaçamento $4 \times 2 \mathrm{~m}$.

\section{REFERÊNCIAS BIBLIOGRÁFICAS}

Anghinoni, I.; Salet, R. L. (1998). Amostragem do solo e as recomendações de adubação e calagem no sistema plantio direto. In: Nuernberg, N.J. (Ed.) Conceitos e fundamentos do sistema de plantio direto. Lages: Sociedade Brasileira de Ciência do Solo. p. 27-52.

BARBER, S.A. Soil nutrient bioavailability: A mechanistic approach. New York, Jonh Wiley \& Sons, 1984. 398p.

Barros, N. F.; Neves, J. C. L.; Novais, R. F. (2005). Fertilidade de solos, nutrientes e produção florestal.

Visão Agrícola.4: 76-79. 
Brienza Júnior, S.; Oliveira, R. P.; Denich, M.; Vlek, P. L. G. (2011). Plantio de árvores de crescimento rápido para recuperação de áreas agrícolas na Amazônia Oriental brasileira: avaliações de sobrevivência e produções de milho e mandioca. Pesquisa Florestal Brasileira. 31(68): 347-353.

Carvalho, J.R.P; Silveira, P.M.; Vieira, S.R. (2002). Geostatistics to determine spatial variability of soil chemical properties using different preparation systems. Pesquisa Agropecuária Brasileira, v.37, n. 8, p.1151- 1159 .

DeMaria, I.C.; Nnabude, P.C. \& Castro, O.M. (1999) Longterm tillage and crop rotation effects on soil chemical proprieties of a Rholic Ferrasol in southern Brazil. Soil Tillage Res., 51:71-79.

Falcão, F.; Escosteguy, P. A. V. (2006). Variação de matéria orgânica, fósforo, potássio e enxofre, em função da profundidade de amostragem e do relevo, de um Latossolo com sistema plantio direto consolidado. In: VI Reunião Sul-brasileira de Ciência do Solo, Fertilidade do solo - (Re) emergindo sistêmica. Passo Fundo, RS: SBCS - NRS.

Foloni, J. S. S.; Rosolem, C. A.; Calonego, J. C.; Garcia, R. A. (2012). Balanço de Potássio no Sistema Plantio Direto em Razão da Adubação Potássica na Sucessão Milheto-Soja. In: Congresso Brasileiro de Soja. Soja: integração nacional e desenvolvimento sustentável: resumos. Brasília, DF: Embrapa. 197 p.

Fundação Amazônia de Amparo a Estudos e Pesquisas (FAPESPA). (2016). Estatística municipal: Dom Eliseu. Diretoria de Estatística e de Tecnologia e Gestão da Informação - Belém.

Grant, C. A.; Flaten, D. N.; Tomasiewicz, D. J.;Sheppard, S. C. (2001). A importância do fósforo no desenvolvimento inicial da planta. Informações Agronômicas. 95, p. 1-5.
Klein, C.; Agne, S. A. A. (2012). Fósforo: de nutriente à poluente! Revista Eletrônica em Gestão, Educação e Tecnologia Ambiental. 8(8): 17131721.

Klepker, D. \& Anghinoni, I. Crescimento radicular e aéreo do milho em vasos em função do nível de fósforo no solo e da localização do adubo fosfatado. R. Bras. Ci. Solo, 19:403-408, 1995.

Laclau, J.-P., Ranger, J., Gonçalves, J. L. M., Maquère, V., Krushe, A. V., Thongo M'Bou, A., et al. (2010). Biogeochemical cycles of nutrients in tropical Eucalyptus plantations. Main features shown by intensive monitoring in Congo and Brazil. For. Ecol. Manag. 259, 1771-1785.

Leite, L. F. C. Biomassa de raízes finas e fertilidade do solo, sob vegetação de caatinga em Caruaru-PE. 1998. 72f. Dissertação (Mestrado em Ciências do Solo). Universidade Federal Rural de Pernambuco UFRPE, Recife, PE, 1988.

Model, N.S. \& Anghinoni, I. Resposta do milho a diferentes modos de aplicação de adubos e técnicas de preparo de solo. R. Bras. Ciência do Solo. 16:5559, 1992.

Nolla, A.; Anghinoni, I. (2006). Atividade e especiação química na solução afetadas pela adição de fósforo em latossolo sob plantio direto em diferentes condições de acidez. Revista Brasileira de Ciência do Solo. 30, p. 955-963.

Novais, R.F.; Smyth, T.J. \& Nunes, F.N. Fósforo. In: Novais, R.F.; Alvarez V., V.H.; Barros, N.F.; Fontes, R.L.F.; Cantarutti, R.B. \& Neves, J.C.L., eds. Fertilidade do solo. Viçosa,MG, Sociedade Brasileira de Ciência do Solo, 2007. v.1. p.471-550.

Pereira, A.R. Biomassa e ciclagem de nutrientes minerais em povoamentos jovens de Eucalyptus grandis e Eucalyptus urophylla, em regiões de 
cerrado. Viçosa, Universidade Federal de Viçosa, 1990. 167p. (Tese de Doutorado)

Rosolem, C. A.; Calonego, J. C.; Foloni, J. S. S. (2003). Lixiviação de potássio da palha de coberturas de solo em função da quantidade de chuva recebida. Revista Brasileira de Ciência do Solo. 27(3): 355362.

Santos, H.P. \& Tomm, G.O. (2003) Disponibilidade de nutrientes e teor de matéria orgânica em função de sistemas de cultivo e de manejo de solo. Ciência Rural. 33:477-486.

Schlindwein, J. A., Anghinoni, I. (2000). Variabilidade Vertical de Fósforo e Potássio Disponíveis e Profundidade de Amostragem do Solo no Sistema Plantio Direto. Ciência Rural. 30(4): 611-617.

Schumacher, M. V. et al. Produção de serapilheira em uma floresta de Araucaria angustifolia (bertol.) kuntze no município de Pinhal Grande-RS. Revista Árvore, v.28, n.1, p.29-37, 2004.

Silva, F. C.; Eira, P. A.; Barreto, W. O.; Pérez, D. V.; Silva, C. A. (1998). Análises químicas para avaliação da fertilidade do solo: Métodos usados na Embrapa Solos. Rio de Janeiro: EMBRAPACNPS. (Documentos, 3).

Silveira, E. R.; Reiner, D. A.; Smaniotto, J. R. (2014). Efeito do espaçamento de plantio na produção de madeira e serapilheira de Eucalyptus dunni na região sudoeste do Paraná. Revista Técnico Científica. 1(2): 1-9.

Sousa, V. G. de. (2011). Comportamento silvicultural e dinâmica de serapilheira em plantios de duas espécies florestais na Amazônia oriental brasileira. $111 \mathrm{f}$. Dissertação (Mestrado) Universidade Federal do Pará, Belém, 2011. 